\title{
Acute Phase Protein Responses in Mice Infected with River Water Contaminated by Pasteurella multocida Type B: 2
}

\author{
${ }^{1,5}$ Mohammed Muqdad Khaleel, ${ }^{1,3}$ Faez Firdaus Jesse Abdullah, \\ ${ }^{1,6}$ Lawan Adamu, ${ }^{1}$ Abdinasir Yusuf Osman, ${ }^{1,3}$ Abdul Wahid Haron, \\ ${ }^{2}$ Mohd Zamri Saad and ${ }^{4}$ Abdul Rahman Omar \\ ${ }^{1}$ Department of Veterinary Clinical Studies, \\ ${ }^{2}$ Department of Veterinary Pathology and Microbiology, \\ Faculty of Veterinary Medicine, Universiti Putra Malaysia, 43400 UPM Serdang, Selangor, Malaysia \\ ${ }^{3}$ Centre for Ruminant Disease, Universiti Putra Malaysia, 43400 UPM Serdang, Selangor, Malaysia \\ ${ }^{4}$ Institute of Bioscience, Universiti putra Malaysia, Malaysia \\ ${ }^{5}$ Department of Microbiology, College of Veterinary Medicine, University of Mosul, Iraq, Mosul \\ ${ }^{6}$ Department of Veterinary Medicine, Faculty of Veterinary Medicine, \\ University of Maiduguri, PMB1069, Borno State, Nigeria
}

Received 2013-08-19, Revised 2013-09-11; Accepted 2013-09-17

\begin{abstract}
Animals are predisposed to infections through varieties of ways which activate the innate immune systems at the initial phase of acute infections. Hemorrhagic Septicemia (HS) is a devastating septicemic disease of cattle and buffaloes caused by a particular serotypes of Pasteurella multocida and serotype B: 2 is the most important cause of the disease in Asia. Therefore, the present study aims to investigate on acute phase protein responses in mice infected with river water contaminated by Pasteurella multocida type B: 2. Five infected mice were placed in each tank containing river water for 24, 48 and $72 \mathrm{~h}$. The groups comprise of five mice each made up of the control, intraperitoneal, oral and the aerosol routes. There were increased concentrations of Haptoglobin $(\mathrm{Hp})$ in the mice inoculated with infected river water kept for $24 \mathrm{~h}$ intraperitoneally $(\mathrm{p}<0.0454)$ relative to the control, oral and the aerosol routes. There was significant increased concentration of Serum Amyloid A (SAA) in the mice that were inoculated with infected river water kept for $72 \mathrm{~h}$ intraperitoneally $(p<0.0020)$ compared to control, orally and the aerosol routes. The intraperitoneal route though most effective in response but practically not feasible for vaccine administration in larger animals and the oral routes was inadequate in eliciting acute phase response in the present study of mice model. Therefore, the aerosol routes could perhaps be a readily available route for effective vaccine administration and heightened immunity in animals considering the progressive responses of APPs through this route.
\end{abstract}

Keywords: Serum Amyloid A (SAA), Haptoglobin (Hp), Pasteurella Multocida type B: 2, Different Routes, Contaminated River Water, Infected Mice Carcasses

\section{INTRODUCTION}

Hemorrhagic Septicemia (HS) is a devastating septicemic disease of cattle and buffaloes caused by fastidious serotypes of Pasteurella multocida (Abdullah et al., 2013a), in Asia serotype B: 2 is the leading cause of HS (Abubakar and Zamri-Saad, 2011) and in some African countries (Abdullah et al., 2013b)

Corresponding Author: Faez Firdaus Jesse Abdullah, Department of Veterinary Clinical Studies, Faculty of Veterinary Medicine, Universiti Putra Malaysia, 43400 UPM Serdang, Selangor, Malaysia Tel: +60386093924 
with high morbidity and mortality leading to huge economic loss (Jesse et al., 2013a).

In recent times, research were conducted to investigate on the survival rate of Pasteurella multocida in river water containing carcasses (Abdullah et al., 2013a). Animals experiencing exogenous or endogenous challenge mount a strong response by activating the innate immune systems at the initial stage of acute infection before the onset of the acquired immune system (Jesse et al., 2013b). The acquired immune system ultimately leads to the development of specific cellular and humoral immune responses. During the preliminary challenge the continued existence of the host depends on the capability of the innate reactions to tussle the causes of disease (Eckersall and Bell, 2010).

The Acute Phase Proteins (APPs) are proteins found in the blood and the circulating concentrations of APPs are correlated to the severity of the infection and hence the concentrations of APPs offers a ready means of assessing the presence and extent of the disease progression (Eckersall and Bell, 2010; Tothova et al., 2013).

However, there are substantial species dissimilarities in the serum concentrations of acute phase proteins (Eckersall and Bell, 2010). Sixfold increase in $\mathrm{Hp}$ concentrations was observed in infected dairy cows and those with metabolic disease compared to animals with minor lesions (Jesse et al., 2013b). Seven and forty fold increase in SAA and Hp were also observed in culled dairy cattle with acute lesions relative to healthy beef animals (Jesse et al., 2013b). Isolation of Pasteurella multocida from calves with respiratory disease was associated with significant increased concentrations of acute phase proteins (Abubakar and Zamri-Saad, 2011).

The infection of mice with infected river water and subsequent alterations of SAA and $\mathrm{Hp}$ in a mice model has not been elaborated in previous studies. This study is a leap in the knowledge of HS transmission in a mice model. Therefore, the present study aims to investigate on acute phase protein responses in mice infected with river water contaminated by Pasteurella multocida type B: 2 .

\section{MATERIALS AND METHODS}

Sixty five healthy male mice of eight to ten weeks old were used in this study. They were obtained from the Institute of Cancer Research (ICR) and kept at the Animal Resource Centre, Universiti Putra Malaysia. The animals were confirmed negative for $P$. multocida following culture of peripheral blood for bacterial isolations, housed in plastic cages and provided with water and pellet ad libitum. Five mice were kept in each plastic cage for the control and treatment groups. The mice were observed for 2 weeks prior to the experiment to make sure that they acclimatize to the environment and were healthy.

\subsection{Inoculums}

Throughout the experiments, the wild-type $P$. multocida B: 2 used in this study were obtained from stock culture. It was isolated from a previous outbreak of HS in the state of Kelantan, Malaysia. Identification of $P$. multocida was made using the Gram-staining method and biochemical characterization of oxidase, urea broth, Sulphur Indole Motility (SIM), Triple Sugar Iron (TSI) and citrate tests. The isolate was confirmed to be $P$. multocida type B: 2 by the Veterinary Research Institute (VRI) Ipoh, Perak. Pure stock culture that was stored on nutrient agar slants was sub-cultured onto $5 \%$ horse blood agar and incubated at $37^{\circ} \mathrm{C}$ for $18 \mathrm{~h}$. A single colony of $P$. multocida was selected and grown in Brain Heart Infusion broth (BHI), incubated in shaker incubator at $37^{\circ} \mathrm{C}$ for $24 \mathrm{~h}$ before the concentration was determined by McFarland Nephelometer Barium Sulfate Standards.

\subsection{Experimental Design in Mouse Model}

The river water was cultured to confirm that it was free from $P$. multocida type B: 2, the river water was obtained from Hulu Langat. Fifteen mice were initially inoculated with $1.0 \mathrm{~mL}$ of $10^{9}$ colony forming unit (cfu) of $P$. multocida type B: 2 intraperitoneally. After 7-8 h of post inoculation survived mice were euthanized by cervical dislocation and the carcasses were placed in a tank containing river water. Five infected mice were placed in each tank for 24, 48 and $72 \mathrm{~h}$. One $\mathrm{ml}$ of the infected river water of $10^{9}$ of Pasteurella multocida type B: 2 were inoculated intraperitoneally and via the aerosol routes while, $0.4 \mathrm{~mL}$ of $10^{9}$ of Pasteurella multocida type B: 2 was inoculated orally into five mice in each group respectively, after $48 \mathrm{~h}$ the mice were euthanized by cervical dislocation. Thereafter, blood samples were collected directly from the heart into plain tubes from the moribund animals to obtain serum for the analysis of serum amyloid A and haptoglobin. The fourth group consists of the control group which had five mice and was inoculated with $1.0 \mathrm{~mL}$ of sterile Phosphate Buffered Saline (PBS) pH7.

\subsection{Acute Phase Proteins (APP) Analysis}

The test kits were highly sensitive, two sites enzyme linked immunoassay (ELISA) obtained from Life Diagnostics Inc. (West Chester) info@lifediagnostics.com and from Tridelta Development Ltd (Ireland) 
info@trideltaltd.com. The APP used in this was mouse serum amyloid $\mathrm{A}$ and mouse haptoglobin.

\subsection{Determination of Mouse Haptoglobin}

Serum samples were used to determine the Haptoglobin (Hp) levels by ELISA technique. Briefly, the microtiter plates were coated with affinity purified anti-Mouse Hp (Life Diagnostics Inc). The mouse Hp standards were prepared as recommended by the manufacturer. Then, $100 \mu \mathrm{L}$ of serum $(1: 10,000$ dilutions) sample was introduced into each well and incubated for 15 min before being washed 4 times with an ELISA washer (BioRad). After that, $100 \mu \mathrm{L}$ of conjugate (1:100 dilutions) was dispersed into the wells and incubated for $15 \mathrm{~min}$ and washed 4 times with the ELISA washer (BioRad). The substrate (Life Diagnostics Inc) was then introduced before incubation at $37^{\circ} \mathrm{C}$. The reaction was stopped by $0.3 \mathrm{M}$ sulfuric acid and the optical density values were measured at absorbance 450 $\mathrm{nm}$ wavelengths in an ELISA Reader (BioRad).

\subsection{Determination of Mouse Serum Amyloid A (SAA)}

Serum samples were used to determine the mouse Serum Amyloid A (SAA) levels by ELISA technique. Briefly, the microtiter plates were coated with affinity purified anti-mouse SAA (Life Diagnostics Inc). The mouse SAA standards were prepared as recommended by the manufacturer. Then, $100 \mu \mathrm{L}$ of serum $(1: 1000$ dilutions) sample was introduced into each well and incubated at $37^{\circ} \mathrm{C}$ for $60 \mathrm{~min}$ before being washed 4 times with an ELISA washer (BioRad). After that, $100 \mu \mathrm{L}$ of conjugate (1:100 dilutions) was dispersed into the wells and incubated at $37^{\circ} \mathrm{C}$ for $30 \mathrm{~min}$ and washed 4 times with the ELISA washer (BioRad). The substrate (Life Diagnostics Inc) was then introduced before incubation at $37^{\circ} \mathrm{C}$. The reaction was stopped by $0.3 \mathrm{M}$ sulfuric acid and the optical density values were measured at absorbance.

\subsection{Statistical Analysis}

All the data's were analyzed using JMP ${ }^{\circledR}$ 9. NC: SAS Institute Inc. software Version. The data were considered significant at $\mathrm{p}<0.05$.

\section{RESULTS}

The concentration of SAA increased significantly $(p<0.0020)$ in the mice that were placed in the infected river water for $72 \mathrm{~h}$ and subsequently inoculated intraperitoneally compared to the control, oral and aerosol routes. There were also significant increased $(p<0.0454)$ in the concentrations of Hp in the group of mice that were placed in the infected river water for $24 \mathrm{~h}$ intraperitoneally relative to the control, oral and the aerosol routes. Figure 1 showed the responses of SAA concentrations in mice post inoculation with river water contaminated with infected mice carcasses for 24, 48 and $72 \mathrm{~h}$ through the different routes of inoculation. Figure 2 presented the responses of $\mathrm{Hp}$ concentrations in mice post inoculation with river water contaminated with infected mice carcasses for 24,48 and $72 \mathrm{~h}$ through the different routes of inoculation.

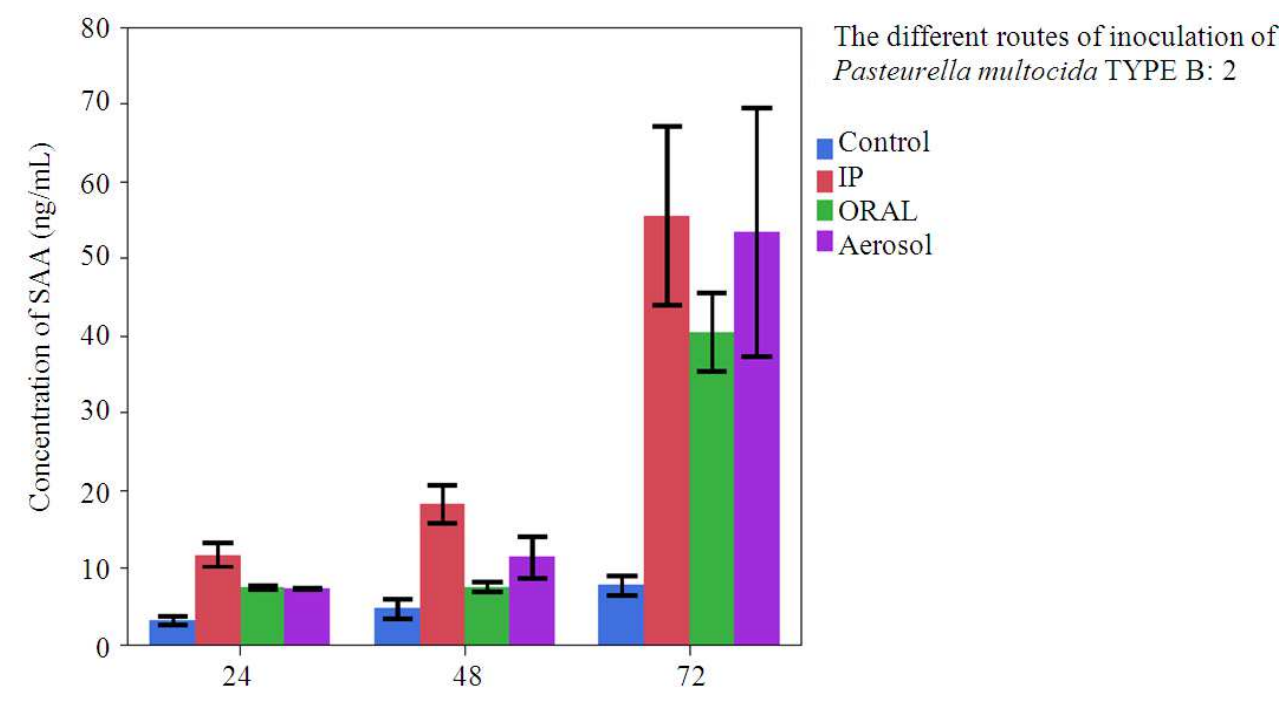

Fig. 1. Responses of SAA in mice post inoculation with river water contaminated with infected mice carcasess for 24,48 and $72 \mathrm{~h}$ throug the different routes of inoculation. SAA = Serum Amyloid A; IP = Intraperitoneal 


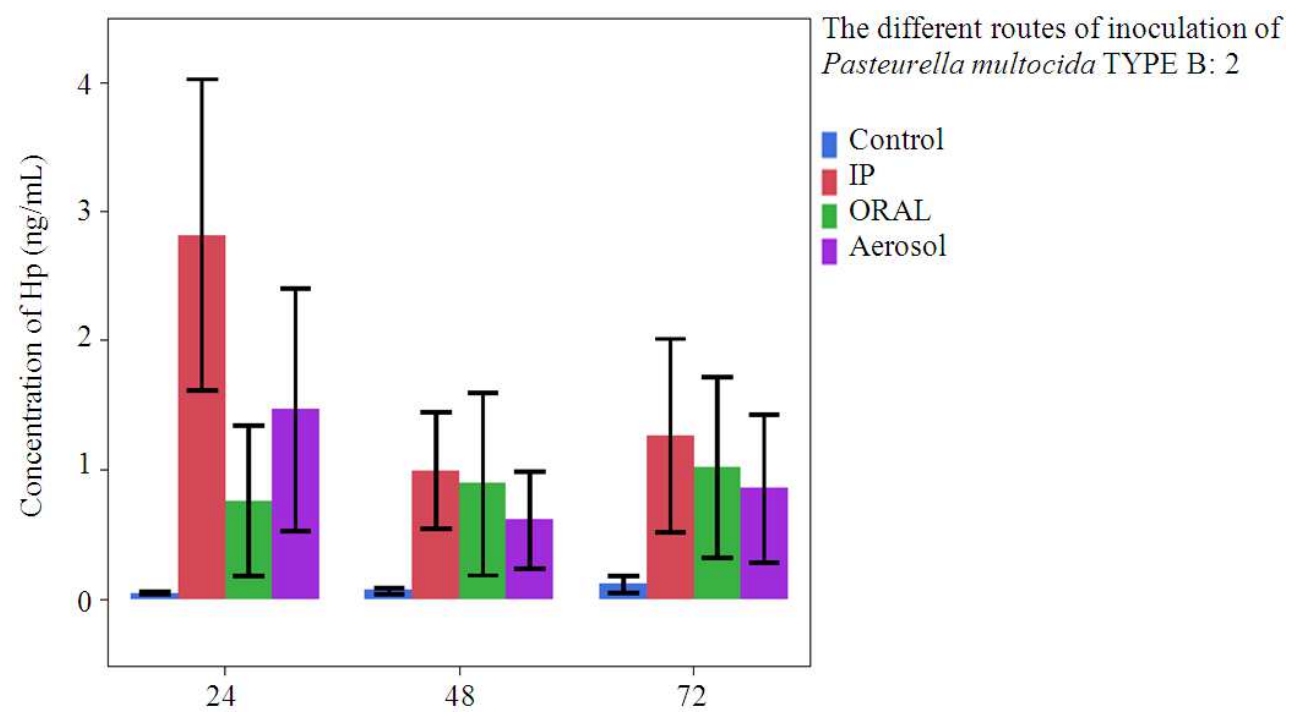

Fig. 2. Responses of $\mathrm{Hp}$ in mice post inoculation with river water contaminated with infected mice carcasess for 24,48 and $72 \mathrm{~h}$ throug the different routes of inoculation. Hp = Haptoglobin; IP = Intraperitoneal

\section{DISCUSSION}

The acute phase response is the first line of immune defense against preliminary infections. The acute phase proteins either increase or decrease in case of infection, inflammation or trauma by greater than 25\% (Abdullah et al., 2013b). In the present study, the intraperitoneal and the aerosol routes proved to be the most effective routes of Pasteurella multocida type B: 2 transmissions in infected river water kept for 24 and $72 \mathrm{~h}$. This was evident by the significant increase in the concentrations of $\mathrm{Hp}$ and SAA through the intraperitoneal and aerosol routes comparative to the oral route, the oral route is the less effective in the transmission of the disease in the present study.

In the present study, there was an increase in the concentration of SAA in the infected river water kept for 72 $h$ via the intraperitoneal and the aerosol routes compared to the oral route. The increase in the concentration of SAA is perchance associated with the inhibitory effect of the inflammatory cells while, the increase in the concentration of $\mathrm{Hp}$ could be related to the bacteriostatic and immunomodulatory effects of reestablishing homeostasis and healing. These findings are in agreement with the study conducted by Cray et al. (2009).

Additionally, in the present study the intraperitoneal and the aerosol routes were the only routes in which the acute phase response were significantly high due to inoculation of the mice with the infected river water kept for 24 and $72 \mathrm{~h}$. The significant increases in the concentrations of $\mathrm{Hp}$ and SAA in the current study could be due to the increased in the production of acute phase proteins by the hepatocytes. The increased rate of infections and stress levels were perhaps due to the pattern of increased cytokines production associated with the infection (Ozkanlar et al., 2012).

In the present study, the concentrations of acute phase proteins particularly $\mathrm{Hp}$ and SAA increase significantly in the infected river water kept for 24 and $72 \mathrm{~h}$ respectively after inoculation. Furthermore, in a study conducted on pigs, the concentration of acute phase proteins increased significantly from 48 to $72 \mathrm{~h}$ (Pomorska-Mol et al., 2013). However, Gomez-Laguna et al. (2010) indicated an increase of acute phase proteins through the intranasal routes challenge by $P$. multocida infection in mice model within $36 \mathrm{~h}$ of post infection.

In another study conducted by Gomez-Laguna et al. (2010) the concentrations of SAA increased significantly $3 \mathrm{~h}$ after both the main and booster intra muscular vaccinations with the wild type P. multocida B: 2 . Calves vaccinated via the intra muscular route developed strong immunity to wild-type of P. multocida B: 2 . However, the serum SAA concentrations of intra nasal vaccinated and control calves increased significantly.

Furthermore, Abdullah et al. (2013b) observed significant increase of 7 fold in Hp and less than 3 fold in SAA in calves infected experimentally with $P$. multocida 
type B: 2 and it immunogens. The findings were in accord with the present study in which the mice were inoculated using different routes with $10^{9} \mathrm{CFU}$ of Pasteurella multocida type B: 2 from infected river water kept for 24, 48 and $72 \mathrm{~h}$. The mice became susceptible to the Pasteurella multocida infection in $24 \mathrm{~h}$ which was evident by the significant increase in the concentration of $\mathrm{Hp}$ in the current study.

In the present study, the concentration of $\mathrm{Hp}$ increases sharply in the mice inoculated with the infected river water kept for $24 \mathrm{~h}$ intraperitoneally and by aerosol route. On inoculation with the infected river water kept for $72 \mathrm{~h}$, SAA concentration reaches it peak by the intraperitoneal and aerosol routes. The increases in the concentrations of $\mathrm{Hp}$ and SAA were perhaps associated with the severity of the disease and the quantification of the concentrations could probably proffer a ready means of appraising the presence and extent of the disease progression. These findings were in accord to the findings of (Eckersall and Bell, 2010).

In the present study, mice inoculated through the different routes with the infected river water kept for 48 $\mathrm{h}$ did not show any significant increase in the concentrations of SAA and Hp. The lack of significant increase in the concentrations of SAA and Hp could be associated with the temperature of the water that probably inhibits the growth of Pasteurella multocida (Abdullah et al., 2013a). Additionally in a study conducted by Abdullah et al. (2013a) they found that Pasteurella multocida was not culturable 1 to 14 days after inoculation in water and artificial sea water depending on the mode of storage and temperature. In the present study, the oral route was less afflicted in causing disease state in the mice model. This indicates that the intraperitoneal and aerosol routes could perhaps be a readily available route for effective vaccine administration and heightened immunity in animals.

\section{CONCLUSION}

In conclusion, the findings presented here showed the vulnerabilities of different routes of infection with $P$. multocida type B: 2 . With this model the outcome of disease following identical challenge through the different routes may be possible to distinguish features of $P$. multocida type B: 2 which vary in the transmission rate of the different routes. Additionally, this model could enhance the understanding of the progression of the disease and the various ways for the control of the natural disease.

\section{ACKNOWLEDGEMENT}

We thank the staff of the Department of Veterinary Clinical Studies, Universiti Putra Malaysia and Research Centre for Ruminant Disease, in particular Yap Keng Chee, Mohd Jefri Norsidin and Mohd Fahmi Mashuri for their assistance. The project was funded by Ministry of Higher Education Malaysia.

\section{REFERENCES}

Abdullah, F.F.J., A.Y. Osman, L. Adamu, M.S.M. Yusof and A.R. Omar et al., 2013a. Polymerase chain reaction detection of Pasteurella multocida Type $\mathrm{B}: 2$ in mice following oral inoculation. Asian J. Anim. Vet. Adv., 8: 493-501.

Abdullah, F.F.J., A.Y. Osman, L. Adamu, Z. Zakaria and R. Abdullah et al., 2013b. Acute phase protein profiles in calves following infection with whole cell, lipopolysaccharide and outer membrane protein extracted from Pasteurella multocida type B: 2. Asian J. Anim. Vet. Adv., 8: 655-662. DOI: 10.3923/ajava.2013.655.662

Abubakar, M.S. and M. Zamri-Saad, 2011. Clinicopathological changes in buffalo calves following oral exposure to B: 2. Basic Applied Pathol., 4: 130135. DOI: 10.1111/j.1755-9294.2011.01113.x

Cray, C., J. Zaias and N.H. Altman, 2009. Acute phase response in animals: A review. Comp. Med., 59: 517-526. PMID: 20034426

Eckersall, P.D. and R. Bell, 2010. Acute phase proteins: Biomarkers of infection and inflammation in veterinary medicine. Vet. J., 185: 23-27. DOI: 10.1016/j.tvj1.2010.04.009

Gomez-Laguna, J., F.J. Salguero, F.J. Pallares, M.F.D. Marco and I. Barranco et al. 2010. Acute phase response in porcine reproductive and respiratory syndrome virus infection. Comparative Immunol. Microbiol. Infect. Dis., 33: e51-e58. PMID: 20004019

Jesse, F.F.A., L. Adamu, Y.O. Abdinasir, M.Z. Saad and Z. Zakaria et al., 2013b. Acute phase protein profiles and clinico-pathological changes in mice associated with the infection of Pasteurella multocida type $\mathrm{B}$ and the bacterial lipopolysaccharide and outer membrane protein immunogens. J. Anim. Vet. Adv., 12: 186-193. 
Jesse, F.F.A., L. Adamu, Y.O. Abdinasir, Z. Zakaria and R. Abdullah et al., 2013a. Clinico-pathological responses of calves associated with infection of Pasteurella multocida type B and the bacterial lipopolysaccharide and outer membrane protein immunogens. Int. J. Anim. Vet. Adv., 5: 190-198.

Ozkanlar, Y., M.S. Aktas, O. Kaynar, S. Ozkanlar and E. Kireccl et al., 2012. Bovine respiratory disease in naturally infected calves: Clinical signs, blood gases and cytokine response. Revue Med. Vet., 163: 123130.
Pomorska-Mol, M., I. Markowska-Daniel, K. Kwit, K. Stepniewska and Z. Pejsak, 2013. C-reactive protein, haptoglobin, serum amyloid a and pig major acute phase protein response in pigs simultaneously infected with H1N1 swine influenza virus and Pasteurella multocida. BMC Vet. Res., 9: 14-14. DOI: 10.1186/1746-6148-9-14

Tothova, C., O. Nagy and G. Kovac, 2013. The Use of Acute Phase Proteins as Biomarkers of Diseases in Cattle and Swine. In: Acute Phase Proteins, Janciauskiene, S. (Ed.), Tech Publisher, Rijeka, Croatia. 